\title{
Fundamental Study on Three-dimensional Display Using Water
}

\author{
Kazuma Yoshimuraa, ${ }^{\mathrm{a},}$, Shigeto Nakamura ${ }^{\mathrm{a}}$, Kenichi Iida ${ }^{\mathrm{a}}$ \\ ${ }^{a}$ National Institute of Technology, Nara College, 22 Yata-cho, Yamatokoriyama, Nara, 639-1080, JAPAN \\ *Corresponding Author: kyoshimura@nitnc.net
}

\begin{abstract}
Holograms and computer graphics (CG) are used for presenting three-dimensional shapes. However, these are based on video technology and cannot actually form an object. Therefore, we develop a display device using a water column formed by water jet. By using a liquid, the displayed object can be changed to an arbitrary shape and one can touch those objects. By increasing the number of nozzles, the device can reproduce the object in details.

However, the number of nozzles that can be used depends on the performance of the pump. If the outflow shape can be changed for each nozzle, various displays can be made. In this paper, we numerically analyze the shape of the outflow formed by the water jet of the nozzle.
\end{abstract}

Keywords: Three-dimensional display, Water column control, Numerical analysis.

\section{Introduction}

Recent years, there are many methods of displaying three-dimensional shapes using image techniques such as holograms, CG, and so on. However, the presented objects cannot be touched. It is only displayed virtually. The display devices that actually represent shapes are at the research stage. In this research, we focus on the property of the liquid that the shape can be arbitrarily deformed and we consider to develop a display device using water. Water has many advantages, for instance, being able to provide multi-sensory information such as touch and temperature. In this research, we will work on the development of the three-dimensional shape display using water.

As a display using water, there is a fog screen ${ }^{(1)}$ that projects light to fog with a projector. By artificially generating fog, the two-dimensional image output from the projector is irradiated to the water droplets arranged in a plane and converted to three dimensions. Also, fog display that applies this technology has been developed ${ }^{(1)}$. The problem is that it reflects the observed motion on display and is not a device for representing a three-dimensional shape.

Therefore, in this research, we aim to develop threedimensional display devices using multiple water columns. By adjusting the flow rate using valves and creating a difference in elevation of the water column, it is possible to realize the three-dimensional shape. At present, the number of nozzles that can be used depends on the using pump, and the shape that can be displayed is limited. If a water column with a different outflow shape can be formed for each nozzle, various stereoscopic displays can be expected. Using this device, we will be able to easily present the threedimensional shape regardless of the direction. And because of its touchable nature, it could also be used as a tactile display.

In this paper, we analyze the shape of the outflow formed by the water jet of the nozzle using fluid analysis software ANSYS Fluent and design the nozzle shape. Then, a nozzle fabricates using a $3 \mathrm{D}$ printer, and it is verified whether the expected water column is formed.

\section{Display device}

The display device consists of the tank container, the hose for forming a water column, and the circulation pump. The schematic of this device is shown in Fig. 1 and the built experimental device is shown in Fig. 2. The water tank is made of acrylic and measuring $300 \mathrm{~W} \times 200 \mathrm{D} \times 250 \mathrm{H} \mathrm{mm}$. A branch nipple is used as a hose to form a water column, and it is connected to a pump with a flow rate of $27 \mathrm{~L} / \mathrm{min}$. The nozzle is placed in the display unit, and experiments are performed. 


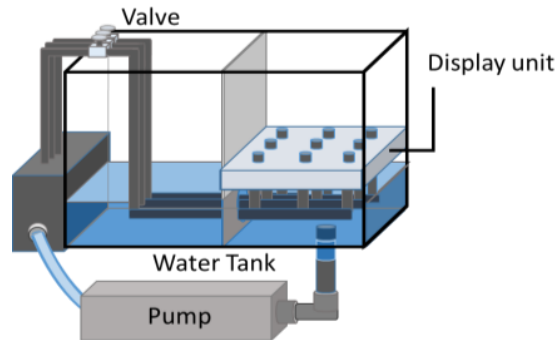

Fig. 1. Schematic diagram of 3D-display.

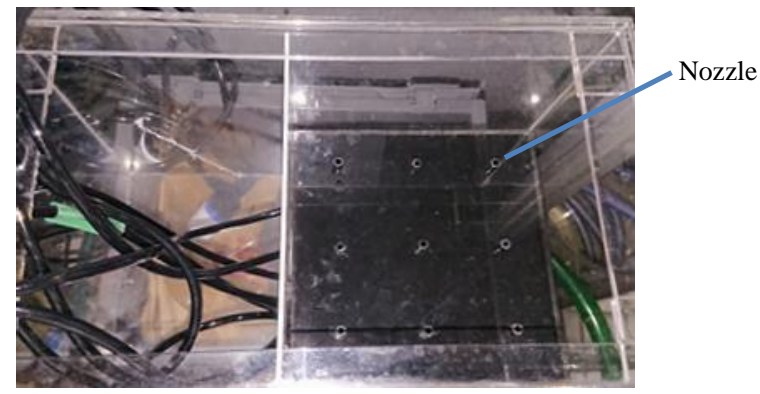

Fig. 2. Schematic diagram of experimental device.

\section{Liquid outflow shape}

Fig. 3 shows three types of outflow shapes. In this study, we aim to create straight, round, and rectangle outflow shapes. The outflow shape of the rectangle nozzle is shown in Fig.4. On the other hand, the nozzle that forms a round shape has not realized yet. Thus, we analyze a cylindrical nozzle that forms a cylindrical water column.

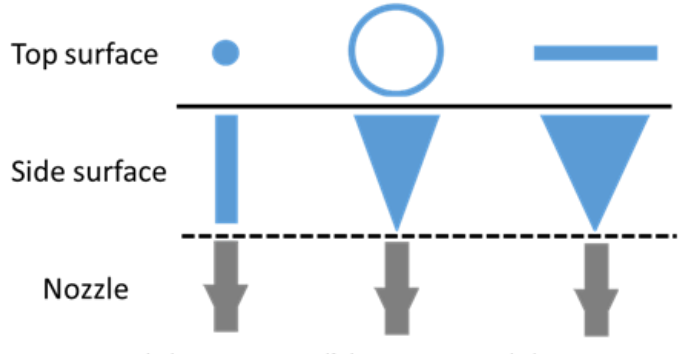

(a) Straight (b) Round (c) Rectangle

Fig. 3. Outflow shape.

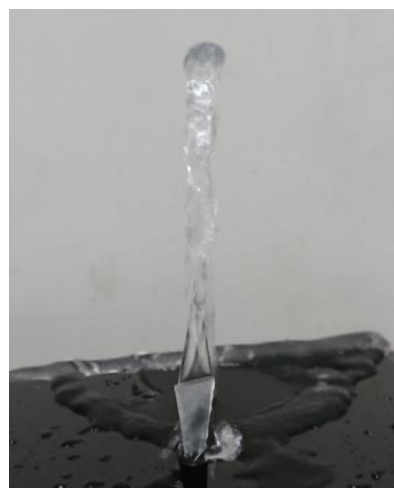

Fig. 4. Outflow shape of rectangle nozzle.
The surface tension of water may affect the outflow shape. Therefore, we analyze two nozzles. Flow analysis of nozzles with a cylindrical inner diameter of $3 \mathrm{~mm}$ (nozzle A) and $7 \mathrm{~mm}$ (nozzle B) is performed. Table 1 shows the dimensions of each nozzle and Fig. 5 shows the nozzle drawing.
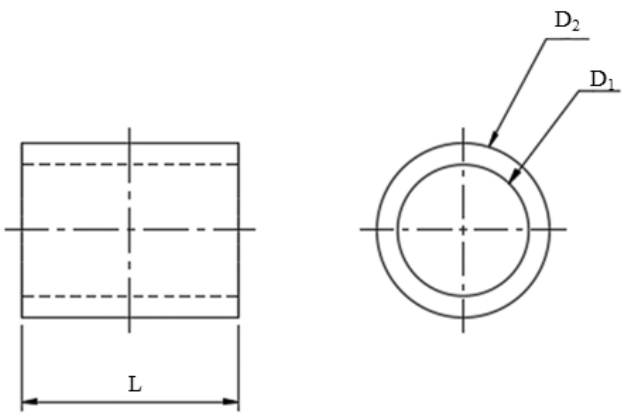

Fig. 5. Nozzle size.

Table 1. Nozzle dimensions.

\begin{tabular}{|c|c|c|}
\hline Nozzle type & A & B \\
\hline $\mathrm{L}[\mathrm{mm}]$ & 5 & 5 \\
\hline $\mathrm{D}_{1}[\mathrm{~mm}]$ & 3 & 7 \\
\hline $\mathrm{D}_{2}[\mathrm{~mm}]$ & 4 & 8 \\
\hline
\end{tabular}

\section{Numerical analysis}

\subsection{Calculation area}

The calculation area is created using ANSYS DesignModeler. Table 2 shows the size of the analysis area of each nozzle and Fig. 6 shows the analysis area. As shown in Fig. 5, the nozzle is installed at the bottom of the analysis area.

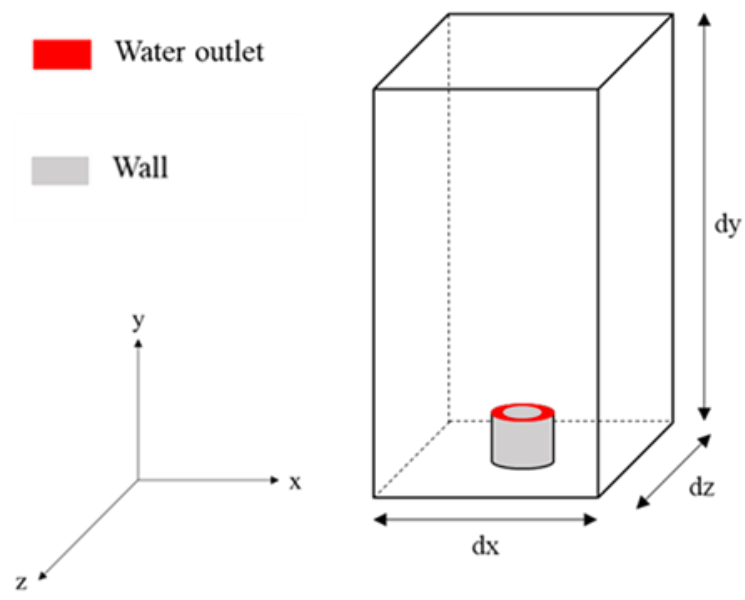

Fig. 6. Calculation area and position of the nozzle. 
Table 4. Simulation conditions.

\begin{tabular}{|c|c|c|}
\hline Nozzle type & A & B \\
\hline Eddy viscosity mode & \multicolumn{2}{|c|}{$k-\varepsilon$} \\
\hline Calculation scheme & \multicolumn{2}{|c|}{ SIMPLE method } \\
\hline Discretization scheme & \multicolumn{2}{|c|}{ Second Order Upwind } \\
\hline Flow rate $\left[\mathrm{m}^{3} / \mathrm{s}\right]$ & \multicolumn{2}{|c|}{$0.013 \times 10^{-3}$} \\
\hline Water velocity $[\mathrm{m} / \mathrm{s}]$ & 2.36 & 1.15 \\
\hline Time step size $[\mathrm{s}]$ & \multicolumn{2}{|c|}{0.0001} \\
\hline Max iterations & \multicolumn{2}{|c|}{20} \\
\hline Number of calculations & \multicolumn{2}{|c|}{1000} \\
\hline
\end{tabular}

\subsection{Calculation grid}

ANSYS Meshing 18.2 was used to generate the calculation grid. Table 3 shows the grid generation conditions.

\subsection{Analytic method}

The fluid analysis software ANSYS Fluent (ver. 18.2) is used to simulate the outflow from the nozzle. The following equations show the governing equations used to analyze this problem.

- Continuous expression

$$
\nabla \cdot \boldsymbol{u}=0
$$

- Navier - Stokes equation

$$
\rho\left(\frac{\partial \boldsymbol{u}}{\partial t}+\boldsymbol{u} \cdot \nabla \boldsymbol{u}\right)=-\nabla p+\mu \nabla^{2} \boldsymbol{u}+\boldsymbol{f}
$$

Here, $\boldsymbol{u}=\{u \mathrm{x}, u \mathrm{y}, u \mathrm{z}\}$ is the velocity of the fluid, $\rho$ is the fluid density, $t$ is the time, $p$ is the pressure, $\mu$ is the viscosity coefficient, and $\boldsymbol{f}=\{f \mathrm{x}, f \mathrm{y}, f \mathrm{z}\}$ is the external force term. Table 4 shows the calculation method. The turbulence model adopts $k-\varepsilon$ and is solved by a SIMPLE method. The flow rate should be $0.013 \times 10^{-3} \mathrm{~m}^{3} / \mathrm{s}$ to match the pump performance.

\subsection{VOF method}

VOF method ${ }^{(2)}$ is one of the approaches to trace the interface of different fluids when simulating multi-phase flow such as water and air. The ratio of the fluid volume to each element in the analysis region is taken as the value $F$, the interface is calculated, and the movement of the water surface is expressed by changing the value of $F$. $F$ takes values from 0 to 1 , and is defined as a gas cell if $F$ is 0 , a fluid cell if $F$ is 1 , and a surface cell if $0<F<1$. An example of the distribution of $F$ value is shown in Fig. 7. In this paper, a free surface is defined as $F=0.5$.

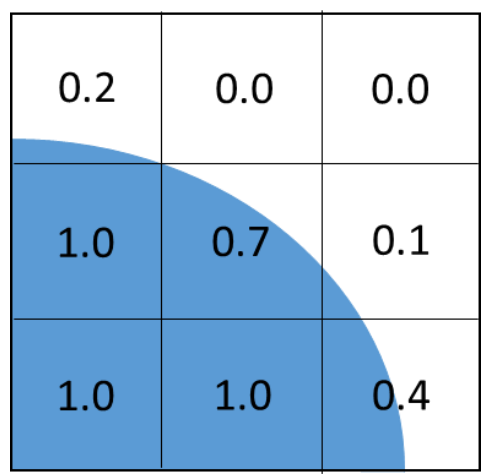

Fig. 7. Distribution example of $F$.

To determine the position of the interface, we solve the following equation with the governing equations (1) and (2).

$$
\frac{\partial \boldsymbol{F}}{\partial t}+\boldsymbol{u} \cdot \nabla \boldsymbol{F}=0
$$

\section{Results and discussion}

\subsection{Analysis of nozzle A}

Fig. 8 shows isosurfaces of volume fraction $(F=0.5)$ at $0.01,0.05$, and 0.10 seconds, and Figs. 9 to 11 show contours of $\mathrm{x}-\mathrm{z}$ planes at 7,8 , and $9 \mathrm{~mm}$ from the bottom at 0.05 and 0.10 seconds. From the results of the contour figure, it can be confirmed that the cylindrical shape is formed at the height of $7 \mathrm{~mm}$, on the other hand, the inside of the water column is filled with water at the height of $8 \mathrm{~mm}$, and the cylindrical shape is not maintained.

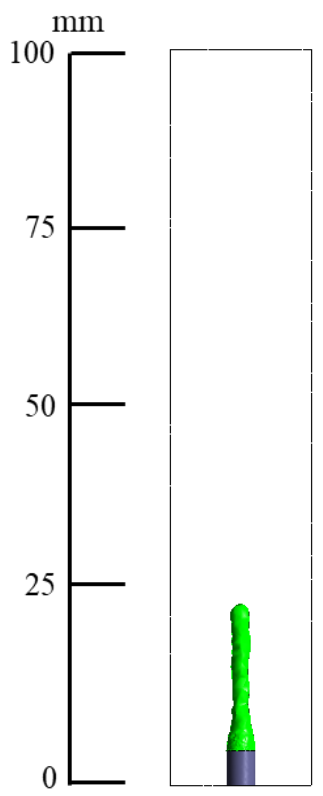

(i ) $0.01 \mathrm{~s}$

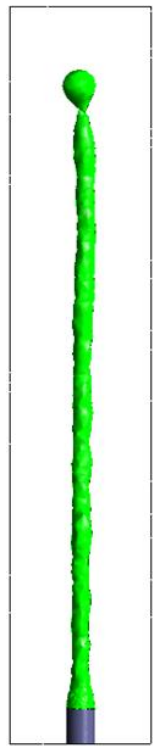

(ii ) $0.05 \mathrm{~s}$

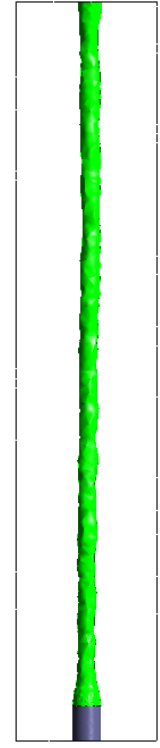

(iii) $0.10 \mathrm{~s}$
Fig. 8. Isosurface of water volume fraction $(F=0.5)$. 
Volume Fraction

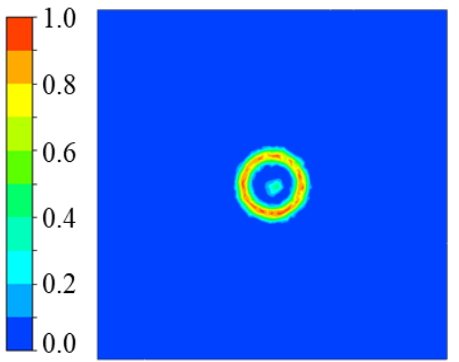

( i ) $0.05 \mathrm{~s}$

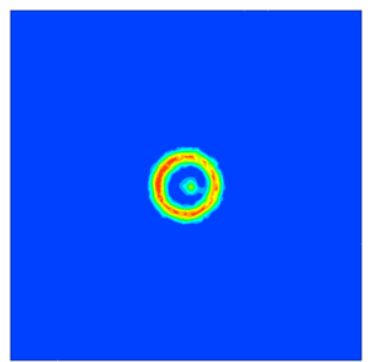

(ii) $0.10 \mathrm{~s}$
Fig. 9. Contour of water volume fraction at $7 \mathrm{~mm}$.

Volume Fraction

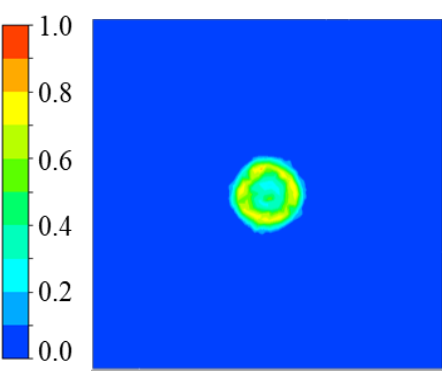

( i ) $0.05 \mathrm{~s}$

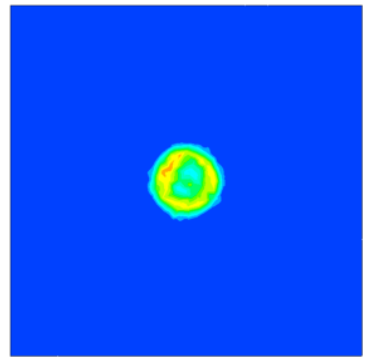

(ii ) $0.10 \mathrm{~s}$
Fig. 10. Contour of water volume fraction at $8 \mathrm{~mm}$.

Volume Fraction

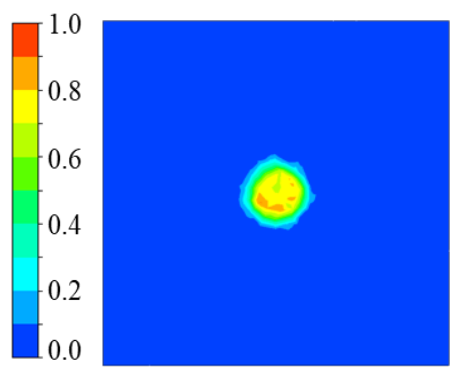

(i ) $0.05 \mathrm{~s}$

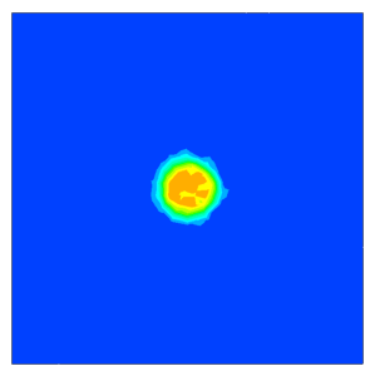

(ii) $0.10 \mathrm{~s}$
Fig. 11. Contour of water volume fraction at $9 \mathrm{~mm}$.

\subsection{Analysis of nozzle B}

Fig. 12 shows isosurfaces of volume fraction $(F=0.5)$ at $0.01,0.05$, and 0.10 seconds, and Figs. 13 to 15 show contours of $x-z$ planes at 7,8 , and $9 \mathrm{~mm}$ from the bottom at 0.05 and 0.10 seconds. From the results of the contour figure, although the cylindrical shape is formed up to the height of $8 \mathrm{~mm}$, it can be confirmed that the inside of the water column is filled with water like the nozzle A at the height of $9 \mathrm{~mm}$ and the cylindrical shape is not maintained.

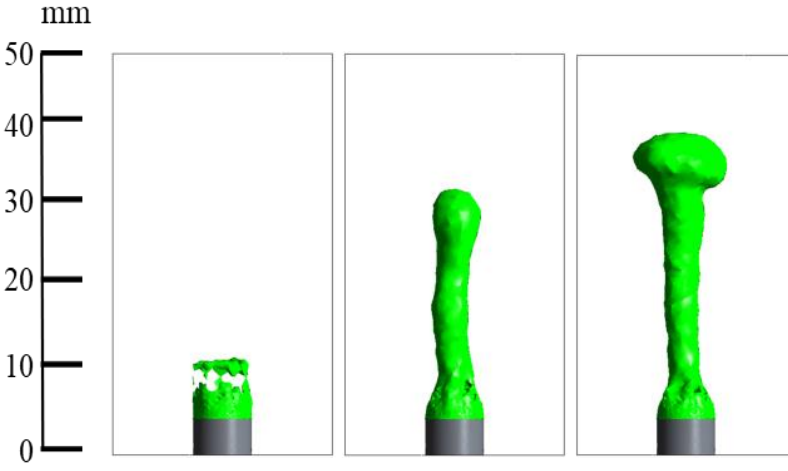
( i ) $0.01 \mathrm{~s}$
(ii ) $0.05 \mathrm{~s}$
(iii) $0.10 \mathrm{~s}$

Fig. 12. Isosurface of water volume fraction $(F=0.5)$.

\section{Volume Fraction}

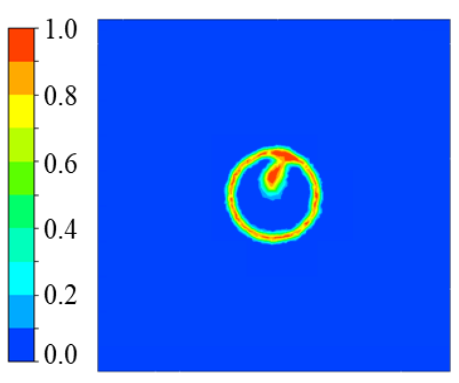

(i ) $0.05 \mathrm{~s}$

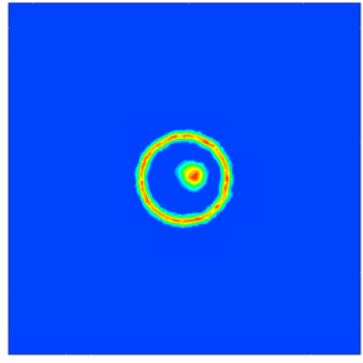

(ii ) $0.10 \mathrm{~s}$
Fig. 13. Contour of water volume fraction at $7 \mathrm{~mm}$.

Volume Fraction

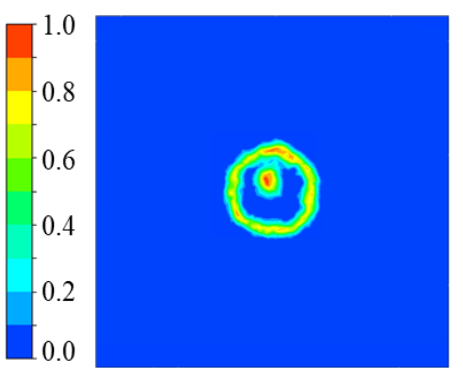

( i ) $0.05 \mathrm{~s}$

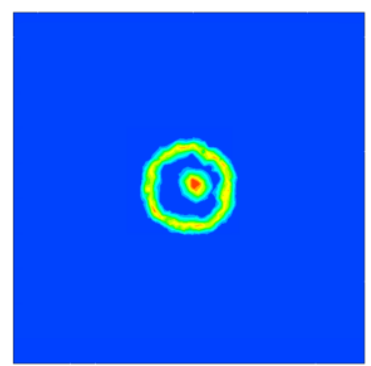

(ii ) $0.10 \mathrm{~s}$
Fig. 14. Contour of water volume fraction at $8 \mathrm{~mm}$.

Volume Fraction

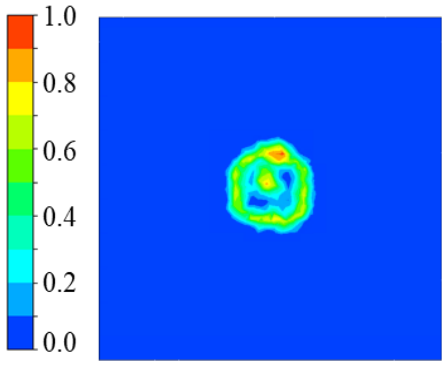

( i ) $0.05 \mathrm{~s}$

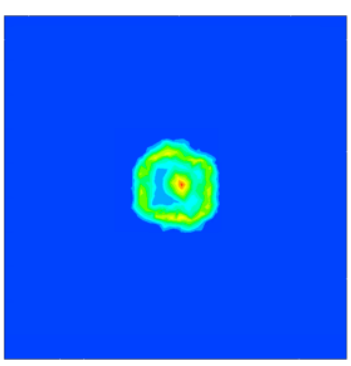

(ii ) $0.10 \mathrm{~s}$
Fig. 15. Contour of water volume fraction at $9 \mathrm{~mm}$. 


\section{Conclusion}

From the results, we have confirmed that the outflow shape cannot be improved simply by increasing the nozzle diameter. It can be seen that water gains mass regardless of the nozzle diameter due to the effect of surface tension. In the future, measures will be taken, such as connecting a guide that guides water to the tip of the nozzle or letting air flow from the center of the nozzle. And based on the analysis results, we create the nozzle and install into the device.

\section{References}

(1) Ismo Rakkolainen, Karri Palovuori: "Interactive Digital FogScreen", Proceedings of the Third Nordic Conference on Human-Computer Interaction, pp. 459460, 2004

(2) B.D.Nichols, C.W.Hirt, and R.S.Hotchkiss, "SOLAVOF: A solution algorithm for transient fluid flow with multiple free boundaries", LA-8355, Los Alamos National Laboratory, 1980 